There were 121 positive cultures positive, $79.3 \%$ in patients obeying the defined criteria and $91.7 \%$ with clinical predictors of positivity. Campylobacter was the most frequently identified agent (68.6\%), followed by Salmonella. Campylobacter decreased within an increasing age whilst Salmonella showed an inverse pattern. Campylobacter was the most frequently identified agent throughout all seasons of the year, followed by Salmonella, except in the winter when Yersinia took the second place. Discussion Sticking to accepted criteria for stool collection and/ or to defined clinical features, increasing the yield of stool cultures.

\section{PO-0132 ACID REFLUX INTO THE OESOPHAGUS AND EXERCISE: A PROSPECTIVE STUDY IN CHILDREN}

C Pacchiarotti, M Barreto, M Campisano, F La Penna, A Bozzone, C Casini, I Caiazzo, A Crescenzi, MP Villa. NESMOS Department, Pediatric Unit Sant'Andrea Hospital, Roma, Italy

\subsection{6/archdischild-2014-307384.796}

Background and aims It has been reported that gastro-oesophageal reflux (GOR) can be induced by exercise, as described in adult subjects; studies in children are lacking. We sought whether the presence of acid in the oesophagus may increase with exercise and its potential relationship with atopy and lung function in children.

Methods We recruited 45 patients (M/F: 30/15) aged $11 \pm 2.7$ years with reported exercise-induced respiratory symptoms; subjects were asked for frequency of gastrointestinal symptoms. All patients did lung function before and after 24-h gastro-oesophageal (GO) $\mathrm{pH}$ monitoring; they also underwent exercise testing (treadmill) before removing GO catheter. GO-pH was also analysed for $6 \mathrm{~min}$ intervals before, during and after exercise. The gastro-oesophageal reflux disease (GORD) was defined as a 24hour reflux index (IR) $\geq 4.5 \%$ and/ or symptom index $\geq 50 \%$. Total serum IgE levels were also assessed.

Results GORD was found in 11/45 (24.4\%) of our patients; these children had also a higher IR score during exercise than patients without GORD (7.1 \pm 18.5 vs $0.5 \pm 2.3, \mathrm{p}<0.05)$. A fall of GO-pH was recorded during exercise, greater in children with GORD as compared with those without $(17.2 \pm 42.2$ vs $0.9 \pm 6.4, \mathrm{p}=0.03)$. The exercise-induced fall in GO-pH was associated with frequent gastrointestinal symptoms and correlated with $\operatorname{IgE}$ levels and baseline $\mathrm{FEV}_{1} \%$ (IgE: $\mathrm{r}=-0.37$, FEV1\%: $r=-0.31, \mathrm{p}<0.05$ for both).

Conclusions Our results suggest that oesophageal acidity increase with exercise, particularly in atopic children with frequent gastrointestinal symptoms and low baseline respiratory function.

\section{P0-0133 GASTROESOPHAGEAL REFLUX IN INFANTS AND OSTEOPHATIC MANIPOLATIVE TREATMENT: AN ALTERNATIVE THERAPY?}

${ }^{1} \mathrm{C}$ Pacchiarotti, ${ }^{2} \mathrm{~F}$ Maioli, ${ }^{1} \mathrm{M}$ Campisano, ${ }^{2} \mathrm{G}$ Addonisio, ${ }^{1} \mathrm{~A}$ Crescenzi, ${ }^{1} \mathrm{~L}$ Brasili, ${ }^{1} \mathrm{G}$ Mastrogiorgio, 'A Macari, 'MP Villa. 'NESMOS Department, Pediatric Unit Sant'Andrea Hospital, Roma, Italy; ${ }^{2}$ NESMOS DEpartment, Osteopathic SChool CERDO, Roma, Italy

\subsection{6/archdischild-2014-307384.797}

Background and aims Several study have been reported that modifying vagus nerve control of transient lower esophageal sphincter relaxation can induce improvement of gastroesophageal reflux disease (GERD). Our aim was to evaluate the efficacy of osteophatic treatment (OMT) in infants with GERD.

Methods We enrolled 40 infants (M/F:24/16), age ranged 1-18 months (median 4 month) attending for persistent reflux. Each patient performed I-GERQ-R questionnaire and ultrasonography of the gastro-esophageal junction before and after treatment. The ultrasound score was 0 to 3 on the basis of severity of reflux (number of reflux episodes in $10 \mathrm{~min}$ ). Moreover each patient did an osteophatic treatment consisting in an extensive physical examination, to evaluate TART parameters ( $\mathrm{T}=$ tissue texture changes; $\mathrm{A}=$ asimmetry; $\mathrm{R}=$ restriction of motion; $\mathrm{T}$ $=$ tenderness). Then, a specific therapeutic intervention was chosen, treating only the parts of the body presenting greater TART modifications.

Results All the somatic dysfunctions observed before OMT (at the scale $32 / 40$ patients, the condyles $36 / 40$ patients, the occipito-mastoid suture $36 / 40$ patients; the stomach $22 / 40$ patients, the small epiploon $30 / 40$ patients) disappeared after treatment. The average score of I GERQ-R questionnaire before and after treatment was $22.7 \pm 4.7$ and $17.2 \pm 4.5$ respectively $(p<$ 0.05). In $29(72.5 \%)$ patients we found an improvement of ultrasound parameters (mean score before and after treatment was $1.7 \pm 0.8$ and $0.7 \pm 0.7$ respectively; $\mathrm{p}<0.05$ ).

Conclusion OMT could be considered as an alternative treatment in infants with gastroesophageal reflux. Further data are needed to confirm our hyphothesis.

\section{PO-0134 COW'S MILK PROTEIN ALLERGY: ORAL FOOD CHALLENGE BEFORE 12 MONTHS OF AGE?}

${ }^{1} \mathrm{C}$ Patraquim, ${ }^{1} \mathrm{~F}$ Neiva, ${ }^{2} \mathrm{H}$ Antunes. ${ }^{1}$ Gastrenterology Hepatology and Nutrition Unit Pediatric Department, Hospital de Braga, Braga, Portugal; ${ }^{2}$ Gastrenterology Hepatology and Nutrition Unit Pediatric Department Life and Health Sciences Research Institute (ICVS) School of Health Sciences University of Minho Braga Portugal and ICVS/3B'S PT Government Associate Laboratory Braga/Guimarães, Hospital de Braga, Braga, Portugal

\subsection{6/archdischild-2014-307384.798}

To characterise the cases of cow's milk protein allergy (CMPA) followed in our GI Outpatient Clinic and determine how much parents save on extensively hydrolyzed milk (EHM) when oral food challenge (OFC) is performed $<12$ months (M).

CMPA patients observed between October2010-October2013 were included in this retrospective study based on Service's CMPA protocol: OFC $>6 \mathrm{M}$, except at parents' request. Calculations: each package of EHM costs $20 €$ and has 102 doses and of infant formula (IF) costs $10 €$ and has 181 doses, according to each infant's nutritional needs. Statistical analysis: Mann-Whitney test.

Sixty-four children were included; 59\% females. The median age of onset was $3 \mathrm{M}(0.43-12 \mathrm{M})$. Most frequent symptoms were cutaneous $(n=37)$ and gastrointestinal $(n=27)$. IF was the main dairy product that triggered symptoms $(64 \% ; 22 \%$ with hypoallergenic formula). Family history of atopy was positive in $63 \% ; 54 \%$ had atopic disease, namely atopic dermatitis (82\%). Measurement of cow's milk-specific IgE was performed in $33 ; 24$ with positive results. Fifteen of 20 children with information about the use of IF in maternity had the first dose of CMP at that time. The first OFC was positive in $45 \%$, and was performed at a median age of $10 \mathrm{M}$ (3-39 M). Forty children had their OFC $<12 \mathrm{M}$ and in most cases $(\mathrm{n}=25)$ it was negative. Performing the OFC $<12 \mathrm{M}$, each infant with a negative result spared $326 €$ on EHM. Tolerance was achieved at a median age 